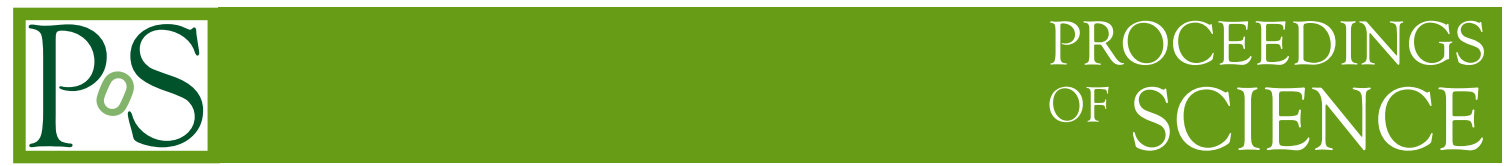

\title{
Jet reconstruction with first data in ATLAS
}

\author{
Damir Lelas on behalf of the ATLAS Collaboration* \\ University of Victoria, Victoria, BC, Canada \\ E-mail: damir.lelas@cern.ch
}

\begin{abstract}
The algorithms used for jet reconstruction in ATLAS are presented. General performance aspects like jet signal linearity and the achievable jet energy resolution are discussed. Strategies and concepts of the jet calibration from the basic calorimeter signals to particle level will be outlined, including aspects of relative and absolute corrections derived from in-situ calibration channels accessible in first data, like QCD dijets and direct photon production.
\end{abstract}

2008 Physics at LHC

September 29 - 4 October 2008

Split, Croatia

* Speaker. 


\section{Introduction}

High quality and highly efficient jet reconstruction is an important tool for almost all physics analyses to be performed with the ATLAS experiment at the Large Hadron Collider (LHC) at CERN, e.g. top-quark and standard model physics as well as searches beyond the standard model.The top mass measurements require an uncertainty in jet energy scale of $1 \%$, while QCD inclusive jet cross section measurements require up to $5 \%$ jet scale uncertainty.

\section{Jet algorithms and reconstruction flow for calorimeter jets in ATLAS}

In general, an attempt is made to provide implementations of all relevant jet finding algorithms in ATLAS. These include fixed sized cone algorithms as well as sequential recombination algorithms and an algorithm based on event shape analysis. This approach is a response to the fact that there is no universal jet finder for the hadronic final state in all topologies of interest. The most commonly used jet finder implementations in ATLAS are a seeded fixed cone finder with split and merge, and a $k_{T}$ algorithm [1],[2]. It is anticipated that for the first experimental collision data all implementations of the FASTJET library $\left(k_{T}\right.$, anti- $k_{T}$, Cambridge flavor $k_{T}$ [3]) will be available, as well as the seedless infrared safe cone algorithm SISCONE [4]. Table 1 summarizes the algorithms and configurations which have been used by ATLAS for basically all pre-collision physics studies [5].

The most important detectors for jet reconstruction are the ATLAS calorimeters. For jet finding it is necessary to first combine 200,000 individual calorimeter cell signals into larger signal objects with physically meaningful four-momenta. The two concepts available are calorimeter signal towers and topological cell clusters [6]. Tower signals are on the electromagnetic energy scale while topological clusters are either on this scale or are calibrated on a local hadronic energy scale.

\section{Detector level jet calibration}

The long standing calibration scheme for calorimeter jets in ATLAS is based on cell signal weighting to mostly account for calorimeter non-compensation [5]. It can be applied to both tower and cluster jets, respectively. On top of the global cell-weighting an algorithm dependent jet scale factor is applied. An alternative approach makes use of the longitudinal development of the shower to correct for calorimeter non-compensation. The jet energy is corrected weighting its energy deposits in the longitudinal calorimeter samples. Although the resolution improvement is smaller with respect to other methods, this method is simple and less demanding in terms of agreement between the detector simulation predictions and real data.

The modular concept of local hadronic calibration was also developed in ATLAS. First topological clusters are reconstructed in the calorimeters with an algorithm to optimize noise suppression and particle separation. Shower shapes and other cluster characteristics are then used to classify the clusters as hadronic or electromagnetic. The hadronic clusters are subject to a cell weighting procedure. In contrast to the cell weights mentioned above no minimization is performed and the actual visible and invisible energy deposits in active and inactive calorimeter material as predicted by Monte Carlo simulations are used to derive the weights. One of the advantages of this method 
Table 1: Default jet finder configurations used in ATLAS.

\begin{tabular}{|l|c|c|}
\hline Algorithm & Main parameter & Physics events \\
\hline \hline Seeded fixed cone & $R_{\text {cone }}=0.4$ & $W \rightarrow j j$ in $t \bar{t}$, SUSY \\
(seeded $\left.p_{T}>1 \mathrm{GeV}\right)$ & $R_{\text {cone }}=0.7$ & inclusive jet cross-section, $Z^{\prime} \rightarrow j j$ \\
\hline$k_{T}$ & $R=0.4$ & $W \rightarrow j j$ in $t \bar{t}$, SUSY \\
& $R=0.6$ & inclusive jet cross-section, $Z^{\prime} \rightarrow j j$ \\
\hline
\end{tabular}

is that the jet reconstruction runs over objects which have the proper scale (in contrast to the global approach, where the scale corrections are applied after the jet is reconstructed from uncorrected objects). Further refinements of the jet calibration can be done, for example, using the tracker information: the residual dependence of the jet scale on the jet charged fraction can be accounted for improving the jet resolution.

\section{Jet reconstruction performance: signal linearity and resolution}

In this section the performance in terms of linearity and resolution of different algorithms are compared. The jet corrections are determined using one jet finder configuration and a sample with a perfect detector geometry. One can estimate the shift from a flat response, introduced by the distorted detector, when other configurations and/or a different calorimeter signal basis are considered. This shift can be expressed as the ratio from a given alternative jet reconstruction to the reference from the same jet reconstruction configuration used to derive calibration functions in the ideal detector:

$$
\xi=\frac{E_{\text {jet,calo }}^{\text {alt }} / E_{\text {jet,truth }}^{\text {alt }}}{E_{\text {jet }, \text { calo }}^{\text {ref }} / E_{\text {jet }, \text { truth }}^{\text {ref }}} \approx \frac{E_{\text {jet }, \text { calo }}^{\text {alt }}}{E_{\text {jet }, \text { calo }}^{\text {ref }}}
$$

Figure 1 (left) shows expectations for $\xi$ as function of the jet $p_{T}$ in two different regions of jet rapidity. From this simulation based study one can estimate the sensitivity of a given jet algorithm to the material distortions in the detector. As these distortions include additional inactive material between the electromagnetic liquid argon and the hadronic tile calorimeter (about 10\% increase in nuclear absorption length), the effect is in particular emphasized for low $p_{T}$ jets in the central region. Here the sensitivity to the jet algorithm (seeded cone or $k_{T}$ ), its configuration (narrow or wide jets), and the choice of calorimeter signals (clusters or tower) is also very large.

The jet energy resolution has been evaluated in the distorted detector geometry with the same QCD sample. A typical relative energy resolution achieved without particular corrections for the distorted detector has a stochastic term of about $60 \% / \sqrt{E(\mathrm{GeV})}$ and a high energy limit of about $3 \%$ in the central region of ATLAS. Using QCD dijet simulations, with electronics noise included in the detector simulation but without any pile-up activity, the difference in resolution between tower and cluster jets can be estimated with the test variable $\psi_{\sigma}$, which uses the fractional difference $\Delta_{\sigma}$ in the energy resolution:

$$
\Delta_{\sigma}=\left(\frac{\sigma}{E}\right)_{\text {cluster }}^{2}-\left(\frac{\sigma}{E}\right)_{\text {tower }}^{2}
$$



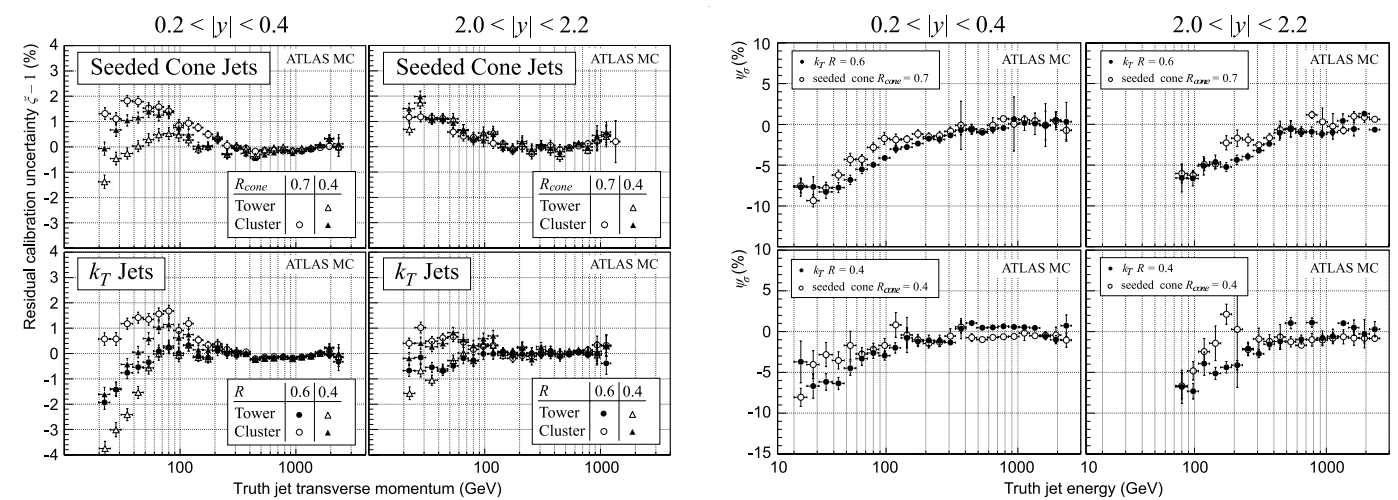

Figure 1: (left) Residual calibration uncertainties for reconstructed jets from various jet finder configurations and the two calorimeter signals, as function of the MC truth jet $p_{T}$ and in two bins of jet rapidity $y$. The reference configuration is seeded fixed cone tower jets with Rcone $=0.7$. The residual calibration uncertainty is given by $\xi$, as defined in Eq. 4.1.

(right) The difference in relative energy resolution $\psi_{\sigma}$ (see Eq. 4.3) between seeded cone cluster and tower jets, and $k_{T}$ cluster and tower jets, respectively, as function of the matching particle jet energy, and in two different regions of jet rapidity $y$. A negative value for $\psi_{\sigma}$ indicates a better resolution for cluster jets.

$\psi_{\sigma}$ can then be defined as:

$$
\psi_{\sigma}=\sqrt{\Delta_{\sigma}} \quad\left(\text { for } \Delta_{\sigma}>0\right) \quad \text { and } \quad-\sqrt{-\Delta_{\sigma}} \quad\left(\text { for } \Delta_{\sigma}<0\right)
$$

Figure 1 (right) shows the prediction for $\psi_{\sigma}$ for various jet configurations in two different kinematic regimes defined by the jet rapidity. The effect of noise suppression implicit for cluster jets is particularly visible at low energies and for wider jets, where more calorimeter cells with noise contribute to the tower jets than in narrow jets.

\section{In-situ calibration strategies with first data in ATLAS}

The validation of the whole jet calibration has to be performed in-situ using suitable physics processes. The validation procedure will start with QCD dijet events, which allows a check of the uniformity of the calibration as a function of azimuth $\phi$ and of pseudo-rapidity $\eta$. The uniformity in $\phi$ can be checked by studying jet rates; the uniformity in $\eta$ can be validated using $p_{T}$ balance between the jets. After a uniform detector response is obtained, the absolute hadronic energy scale will be studied using $\gamma$ or $Z+$ jet events, in which the $Z$ boson is reconstructed via the $Z \rightarrow e^{+} e^{-}$ or $\mu^{+} \mu^{-}$decay. The $p_{T}$ balance between the jet and the boson in such events will be used to relate the hadronic scale of the jets to the well understood energy of electromagnetic objects. The missing $E_{T}$ projection method is an alternative approach in ATLAS to test the jet energy scale.

Figure 2 (left) shows the integrated luminosity required to reach a statistical precision of $0.5 \%$ of the $p_{T}$ dijet balance fit mean value in the probe range $0.7<\eta<0.8$, where $0<\eta<0.7$ is taken as the reference region. A $0.5 \%$ precision can be reached in this $\eta$ region with $10 \mathrm{pb}^{-1}$ of data for $p_{T}<300 \mathrm{GeV}$, and with $100 \mathrm{pb}^{-1}$ for $p_{T}<500 \mathrm{GeV}$. 

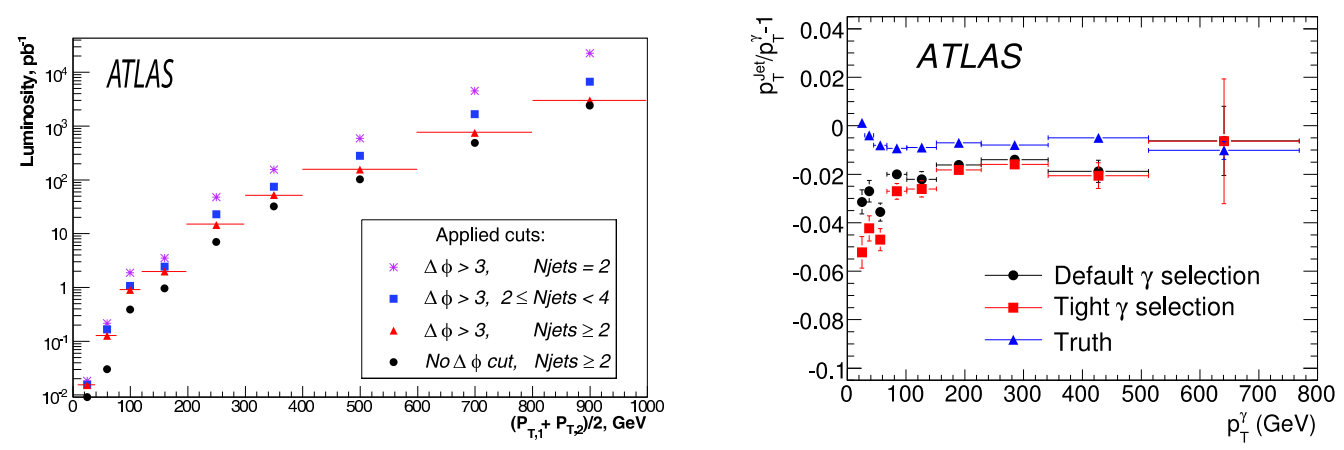

Figure 2: (left) Integrated luminosity required to reach $0.5 \%$ precision for various $p_{T}$ ranges in the region $0.7<\eta<0.8$ with different sets of selection cuts: all PYTHIA dijet events (circles), requiring $\Delta \phi>3$ between the two leading jets (triangles), requiring in addition less than 4 reconstructed jets in an event (squares), requiring exactly two reconstructed jets (stars). The reference region is $0<\eta<0.7$.

(right) The most probable value of the $\gamma$-jet balance at reconstruction level for cone jets with $R=0.7$. Circles and squares are for default and tight selection, respectively, and the triangles show the truth level balance. The back-to-back $\Delta \phi$ cut is applied.

Figure 2 (right) shows the measured $\gamma$-jet $p_{T}$ balance. The photon $p_{T}$ is used as the reference and the photon and the jet are required to be back-to-back in $\phi$. The $p_{T}$ balance above $80 \mathrm{GeV}$ is flattening at the level of -0.02 . At low $p_{T}$ the balance with the tight photon selection is a few percent below the balance measured with the default cuts. The photon reconstruction efficiency is low, and the stringent isolation cuts likely bias the sample composition rejecting events with strong ISR or underlying event [5]. A $100 \mathrm{pb}^{-1}$ of luminosity is sufficient to reach a precision of $1-2 \%$ for jets in the range $300<p_{T}<500 \mathrm{GeV}$. One can expect that the threshold of the un-prescaled photon trigger will be $60 \mathrm{GeV}$ for early running and prescale factors will have to be applied for lower $p_{T}$. There is a non negligible background coming from dijet events.

\section{References}

[1] S. Catani, Y.L. Dokshitzer, B.R. Webber, Phys. Lett.B285 (1992) 291-299.

[2] S.D. Ellis and D.E. Soper, Phys. Rev. D48 (1993) 3160-3166.

[3] Y.L. Dokshitzer, G.D. Leder, S. Moretti, B.R. Weber, JHEP 08 (1997) 001.

[4] G.P. Salam and G. Soyez, JHEP 05 (2007) 086.

[5] ATLAS Collaboration, Expected Performance of the ATLAS Experiment, Detector, Trigger and Physics, CERN-OPEN-2008-020, Geneva, 2008, to appear.

[6] W. Lampl et al, Calorimeter Clustering Algorithms: Description and Performance, ATLAS Liquid Argon Public Note, ATL-LARG-PUB-2008-002, 2008. 